\title{
Preparation of High Purity Trimethylborane ${ }^{1}$
}

\author{
Gaylon S. Ross, Delmo Enagonio, Clifford A. Hewitt, and Augustus R. Glasgow
}

(October 2, 1961)

\begin{abstract}
The preparation of high-purity trimethylborane is described. Impurities were removed by fractionation of the ammonia adduct, $\mathrm{NH}_{3}: \mathrm{B}\left(\mathrm{CH}_{3}\right)_{3}$. Mass spectrometric analyses were used during the fractionation to determine these impurities. After purification of the $\mathrm{B}\left(\mathrm{CH}_{3}\right)_{3}$ in the form of its ammonia adduct, high-purity hydrogen chloride was used to regenerate the $\mathrm{B}\left(\mathrm{CH}_{3}\right)_{3}$. This reaction and the subsequent distillation were carried out in a stainless steel system. Storage as a gas in stainless steel cylinders, rather than as a solid addition complex in glass vessels, eliminated the possibility of recontamination on regeneration. The purity of $\mathrm{B}\left(\mathrm{CH}_{3}\right)_{3}$ was determined cryoscopically to be 99.9 mole percent.
\end{abstract}

\section{Introduction}

Trimethylborane, $\mathrm{B}\left(\mathrm{CH}_{3}\right)_{3}$, is one of a series of boron compounds which have been prepared in high purity for use in the calorimetric $[1]^{2}$ and spectrometric [2] research programs at the National Bureau of Standards. This highly purified material was successfully used as a neutron counting gas in proportional counters $[3,4]$ after trimethylborane prepared by other methods was found to be unsatisfactory because of impurities.

Trimethylborane is a toxic, reactive gas (bp -20 ${ }^{\circ} \mathrm{C}$ ) which burns spontaneously on contact with air. These properties make purification and storage difficult. The compound is usually prepared in the form of the ammonia adduct, $\mathrm{NH}_{3}: \mathrm{B}\left(\mathrm{CH}_{3}\right)_{3}$, which is subsequently treated with hydrogen chloride to liberate the trimethylborane gas. Inadequate purification of the adduct and further contamination during the reaction with the hydrogen chloride produces an impure product. In the preparation which is described in detail in this paper, the adduct was rigorously purified and then reacted with highly purified hydrogen chloride. This reaction and the subsequent fractional distillation of the trimethylborane were carried out in a stainless steel system. The product was stored as a liquid in stainless steel cylinders. Several hundred grams of trimethylborane were prepared, purified, and stored. The material was found to have a purity greater than 99.9 mole percent. This sample was used to establish reliable values for a large number of physical properties.

1 Presented at the 128th meeting of the American Chemical Society at Minne. apolis, Minn., September 11-16, 1955, before the Division of Industrial and Engineering Chemistry.

${ }^{2}$ Figures in brackets indicate the literature references at the end of this paper.

\section{Purification of the Reactants}

\subsection{Ammonia Addition Compound}

The ammonia addition compound of trimethylborane is a white crystalline solid that decomposes slowly in the presence of air and water vapor. All operations were therefore performed in sealed glass systems with the material under its own dissociation pressure. Two lots of the ammonia adduct, received from different sources, ${ }^{3,4}$ were used as starting material. The trimethylborane in these lots had been prepared from a methyl Grignard reagent and boron trifluoride in a $n$-butyl ether solution, under a nitrogen atmosphere, by a procedure similar to that described by Brown [6].

The system used in purifying and transferring the ammonia adduct is shown in figure 1. All parts of the glass system, except the glass ampoule containing the addition compound, were "flamed" to facilitate drying; the entire system was then evacuated to a pressure of $10^{-5} \mathrm{~mm} \mathrm{Hg}$. In the first lots processed, ampoules containing the original sample (D in fig. 1) were not equipped with break-off tips. In this case a scratch was filed on the narrowed neck of the ampoule at $\mathrm{A}$ so that it could be broken by the hammer B. The ampoule was attached to the transfer train at $\mathrm{C}$ by means of Apiezon wax W. Regular break-off tip ampoules were used in the second lots processed.

Fractionation of the ammonia adduct yielded six fractions as follows:

Fraction I-The addition compound in the ampoule D was cooled to approximately $-78{ }^{\circ} \mathrm{C}$ and

3 Robert C Petry and Frank Verhoek, Ohio State Research Foundation Columbus, Ohio, supplied $280 \mathrm{~g}$ for use in the calorimetric and spectrometric studies of trimethylborane at the National Bureau of Standards.

studies of trimethylborane at the National Bureau of Standards. ${ }^{4}$ G. A. Ferguson, Jr., Naval Research Laboratories, Washington 25, D.C.
supplied 282 g. The trimethylborane derived from this quantity of adduct was used as a neutron counting gas in proportional counters at the Naval Research Laboratories. 


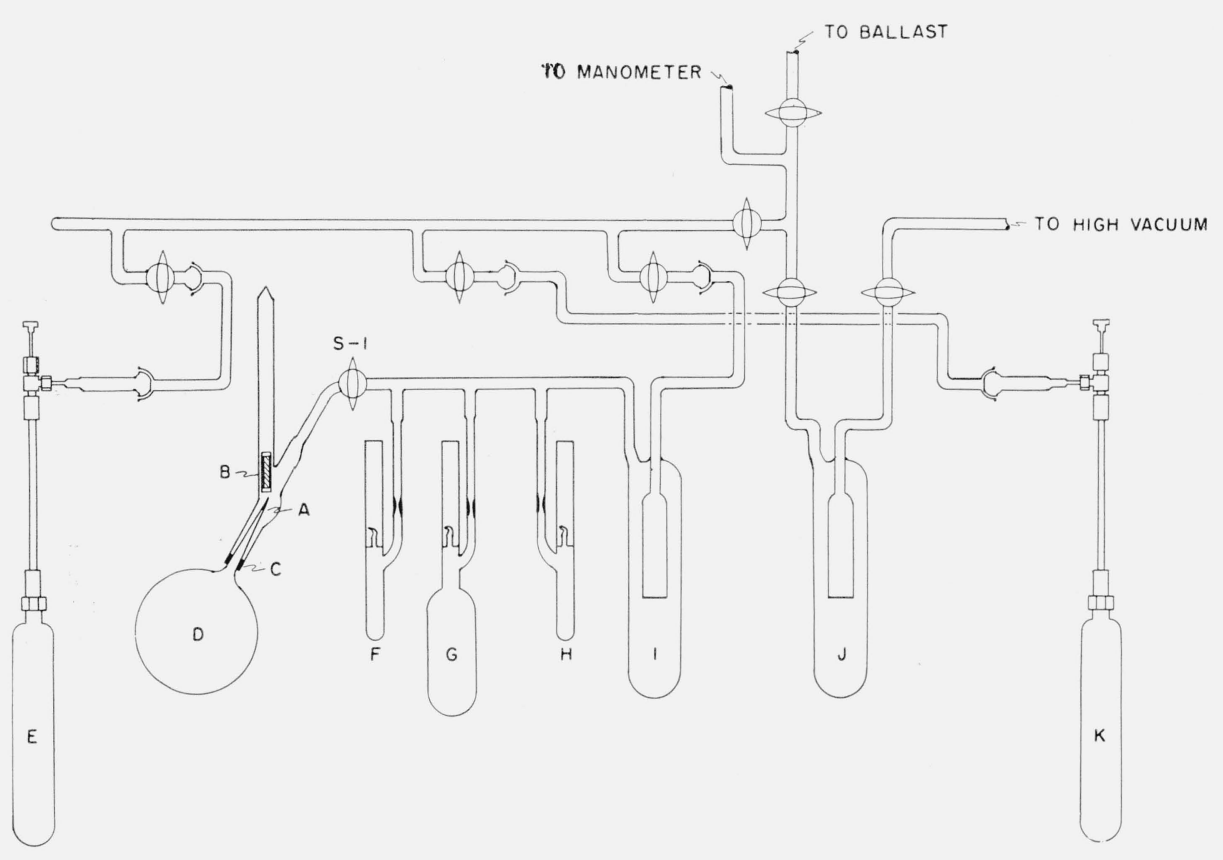

Figure 1. Apparatus for purifying ammonia trimethylborane.

then the tip of the ampoule was broken. The vapor phase existing above the sample at this temperature was collected in cylinder $\mathrm{E}$, which was cooled with a liquid nitrogen bath. Mass spectrometric analysis showed this fraction to contain 30 percent air, 15 percent $\mathrm{CCl}_{4}$, and 55 percent $\mathrm{B}\left(\mathrm{CH}_{3}\right)_{3}{ }^{5}$ These impurities could have been introduced during the original preparation or transfer because carbon tetrachloride was used to clean the system.

Fraction $I I$-The material remaining in ampoule $\mathrm{D}$ was heated with a water bath at about $40{ }^{\circ} \mathrm{C}$. The gaseous dissociation products were condensed in traps I and $\mathrm{J}$, which were cooled with solid carbon dioxide and liquid nitrogen, respectively. The contents of this second trap $(\boldsymbol{J})$ were transferred to metal cylinder K. Analysis of this material showed that it contained 60 percent $\mathrm{CH}_{4}, 20$ percent $\mathrm{CH}_{3} \mathrm{OH}$, 5 percent air, 15 percent $\mathrm{Al}\left(\mathrm{CH}_{3}\right)_{3}$. Methane and methanol are logical oxidation and thermal degradation products of trimethylborane. The presence of aluminum trimethyl, while surprising, leads one to suspect that aluminum must have been present in the magnesium used in the preparation of the methyl Grignard reagent. At $-78^{\circ} \mathrm{C}$, the temperature of the first trap I, there is little dissociation of the ammonia adduct, and the ammonia and trimethylborane generated by dissociation of the adduct at $40{ }^{\circ} \mathrm{C}[7]$ were successfully retained by reassociation. Aluminum trimethyl, however, has an appreciable vapor pressure at this temperature and consequently passed on into the liquid nitrogen trap.

Fraction $I I I$-The material retained at $-78{ }^{\circ} \mathrm{C}$ in trap I was then transferred to trap $J$ and subsequently to ampoule G. A sample for analysis was

\footnotetext{
5 These analyses were performed by F. L. Mohler and V. H. Dibeler of thi Bureau and are given in mole percent.
}

collected in ampoule $\mathrm{F}$. In the analysis of the ammonia adduct, the sample was introduced as a gas into the spectrometer. Since ammonia is adsorbed on the walls of the inlet system, only the trimethylborane was detected. For this reason, the results indicated only the presence of trimethylborane rather than ammonia trimethylborane. The material in ampoule $\mathrm{G}$ was sealed and reserved for subsequent reaction with hydrogen chloride in the preparation of trimethylborane.

Fraction $I V$-After removal of fractions I, II, and III, a yellowish solid remained in the original ampoule. This ampoule was heated to $100^{\circ} \mathrm{C}$, and the gaseous products were collected in trap I, which was cooled with liquid nitrogen. After transfer to ampoule $\mathrm{H}$, this material was found to contain $\mathrm{NH}_{2} \mathrm{~B}\left(\mathrm{CH}_{3}\right)_{2}$ (dimethylaminoboron), $\mathrm{B}\left(\mathrm{CH}_{3}\right)_{3}$, and $\mathrm{NH}_{3}$.

Fraction $V$-The glass stopcock, S-1, was then closed and the glass tubing broken to the right of the stopcock. The residue remaining was heated to $150{ }^{\circ} \mathrm{C}$ and the gaseous products were introduced directly into the mass spectrometer. The primary components of this fraction were trimethylborane, ethyl-methyl boron compounds, and an unknown amino boron compound.

Fraction VI-Approximately two percent of the original material remained as a nonvolatile residue. This yellow solid is water-soluble and is presumed to be an organo-boron mixture of unknown composition.

\subsection{Hydrogen Chloride}

Commercial hydrogen chloride gas contains organic impurities and possibly carbon dioxide. The difficulty involved in removing these impurities suggested 


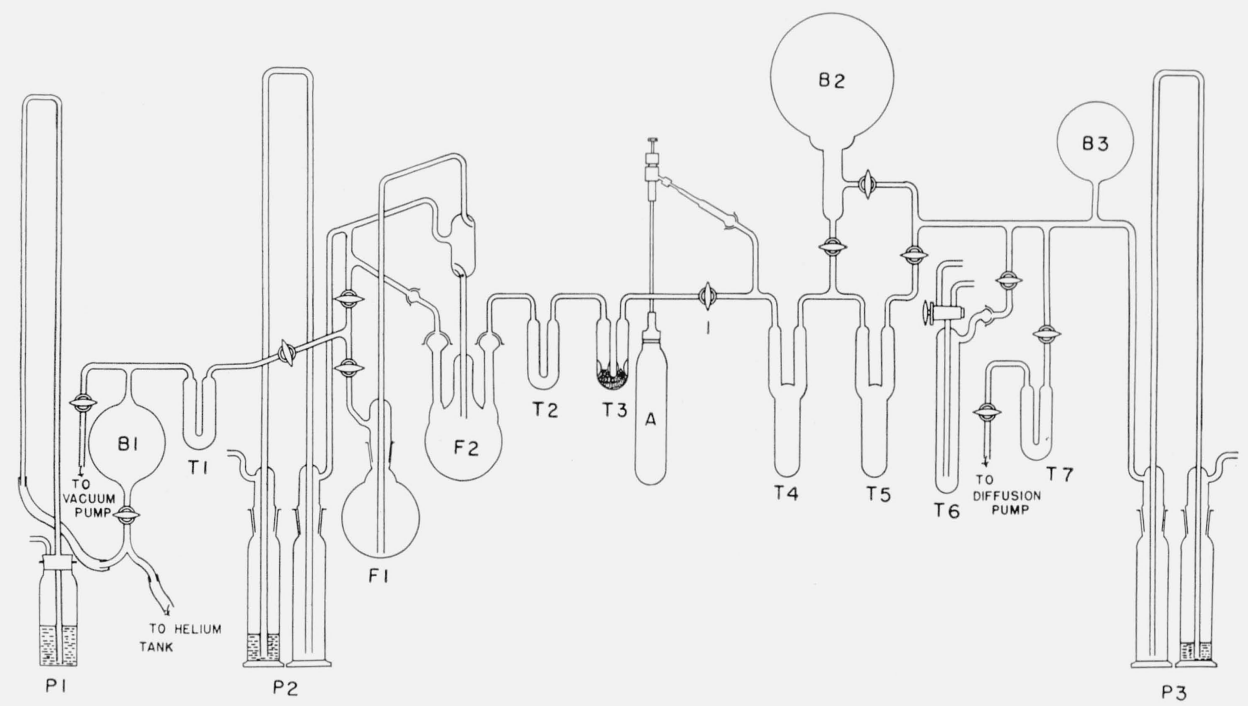

Figure 2. Apparatus for preparing hydrogen chloride gas of high purity.

the preparation of the hydrogen chloride gas by synthesis from purified sulfuric acid and recrystallized sodium chloride. The apparatus for preparing the hydrogen chloride is shown in figure 2. This apparatus was all glass except for the stainless steel cylinder A. This cylinder was one of a group of 3 , all of which had been tested for leaks with a helium leak detector after filling with helium to a pressure of $800 \mathrm{lb} / \mathrm{in}^{2}$. Sulfuric acid was placed in flask $\mathrm{F}-1$ and sodium chloride in $\mathrm{F}-2$. After several flushings with dry helium and subsequent evacuations, a portion of the sulfuric acid was introduced by means of helium pressure into flask $\mathrm{F}-2$. The hydrogen chloride produced was carried along in a continuous stream of dry helium. Pressure regulators $\mathrm{P}-1, \mathrm{P}-2$, and $\mathrm{P}-3$, partially filled with mercury as shown, were used to regulate pressures and flow of the helium and hydrogen chloride, and to act as safety valves.

Traps $\mathrm{T}-2$ and $\mathrm{T}-3$ were cooled to $-78{ }^{\circ} \mathrm{C}$ and trap $\mathrm{T}-4$ to $-196{ }^{\circ} \mathrm{C}$; T-3 was packed with glass wool to retain any solid particles. After being dried in traps $\mathrm{T}-2$ and $\mathrm{T}-3$, the hydrogen chloride gas was condensed in trap T-4. When the reaction was completed, stopcock 1 was closed and the portion of the apparatus to the right of this stopcock was evacuated. This process removed the bulk of the helium present. The residual helium was removed by alternately melting and freezing the hydrogen chloride. The released helium expanded into the ballast bulb B-2 and was removed by pumping through trap $\mathrm{T}-7$, which was cooled to $-196^{\circ} \mathrm{C}$ to prevent fouling of the pumping system with hydrogen chloride. Finally, trap T-4 was warmed to approximately $-120^{\circ} \mathrm{C}$ and the hydrogen chloride transferred to $\mathrm{T}-5$ by cooling this trap to $-196{ }^{\circ} \mathrm{C}$. The hydrogen chloride was transferred back to $\mathrm{T}-4$ and finally collected in the weighed metal cylinder
A. In this manner approximately $450 \mathrm{~g}$ of heliumfree hydrogen chloride was prepared.

\section{Preparation of Trimethylborane}

The addition compound, $\mathrm{NH}_{3}: \mathrm{B}\left(\mathrm{CH}_{3}\right)_{3}$ (fraction III), was reacted with hydrogen chloride in a stainless steel reactor. The reactor and the purification train are shown in figure 3 . The metal cylinders of this train, including the reactor, were filled with helium to a pressure of $380 \mathrm{lb} / \mathrm{in}^{2}$ and tested for leaks with a helium leak detector. Before use, all metal and glass components except joints and valves were heated to about $300{ }^{\circ} \mathrm{C}$ while the system was evacuated. Teflon-packed stainless steel valves of a modified Stimson type [8] and stainless steel connectors were located as shown. Trap T-1 and the section containing the ampoules of ammonia trimethylborane (A-1, 2, 3) were constructed of glass. Steel-to-glass connections were made by the use of a stainless steel male ball-joint and a glass female joint sealed with Apiezon $\mathrm{N}$ grease. The reaction vessel, a $500 \mathrm{ml}$ stainless steel cylinder $\mathrm{C}-3$, and the stainless steel trap T-2 were equipped with reentrant wells $W-1$ and $W-2$ for the insertion of thermocouples. Well W-2 contained three thermocouples: one at the bottom of the reactor, one in the middle of the distillation column $\mathrm{R}$, and one near the top of this column.

The addition compound was treated with hydrogen chloride in the following manner. Batches of from 50 to $150 \mathrm{~g}$ of adduct were transferred from the glass ampoules $A-1$, etc., and deposited as uniformly as possible over the inside surface of the reactor. Total transfer of adduct was considered complete when no solid remained in the glass ampoules, at which time valve 5 was closed. Cylinder $\mathrm{C}-1$ contained a weighed amount of hydrogen 


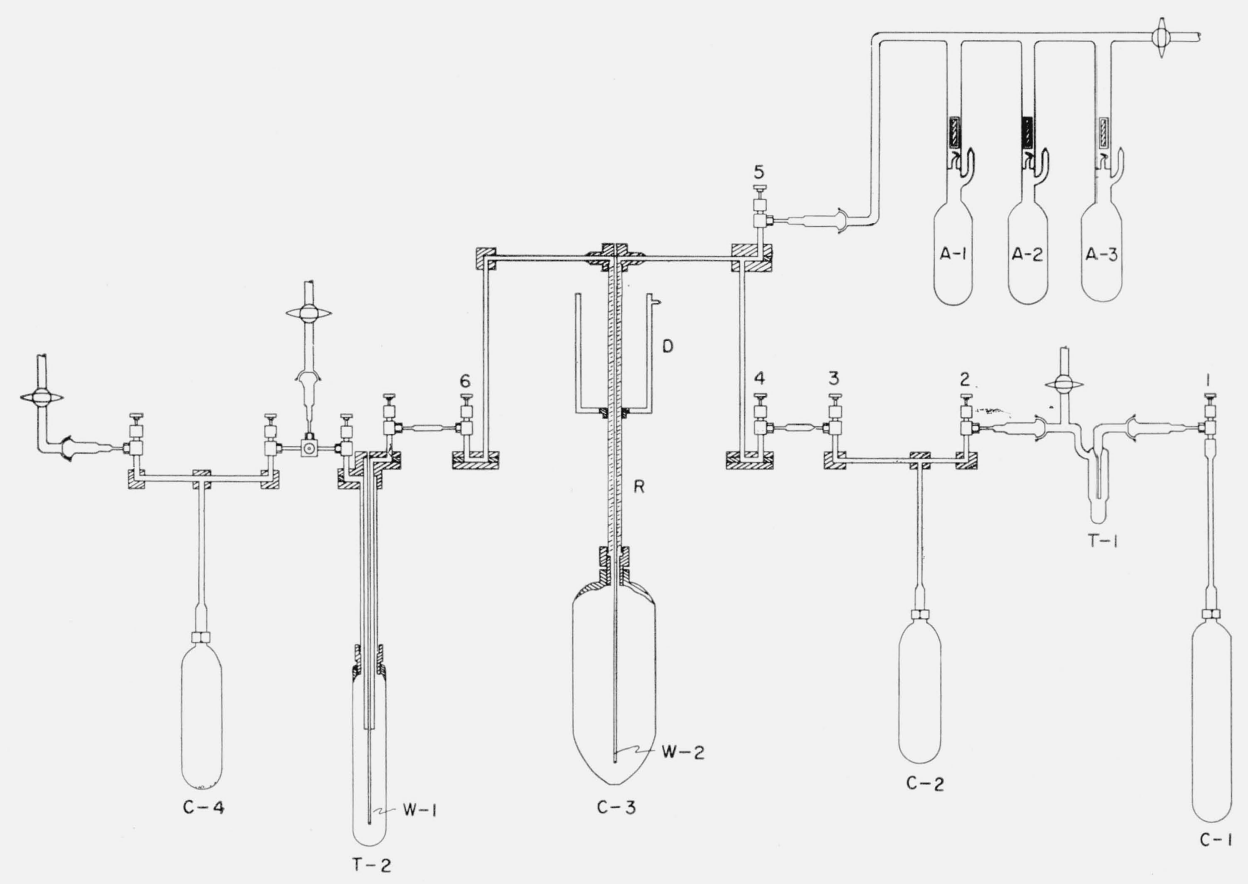

FIGURE 3. Apparatus for preparing and purifying trimethylborane.

chloride sufficient to react with 95 percent of the addition compound used, assuming that all of the hydrogen chloride reacted. Valves 1, 2, 3, and 4 allowed for transfer to or from vessels $\mathrm{C}-1, \mathrm{~T}-1$, $\mathrm{C}-2$, and $\mathrm{C}-3$ and for isolation of parts. Five-gram amounts of hydrogen chloride from cylinder C-1 were condensed in trap $\mathrm{T}-1$, cooled to $-78{ }^{\circ} \mathrm{C}$, and then distilled at this temperature into cylinder $\mathrm{C}-2$ which was cooled by a liquid nitrogen bath. The contents of cylinder $\mathrm{C}-2$, warmed to room temperature, were allowed to react with the adduct in the reactor $\mathrm{C}-3$. The glass trap $\mathrm{T}-1$ allowed one to see the amount of hydrogen chloride condensed in it from cylinder $\mathrm{C}-1$ or recondensed from cylinder $\mathrm{C}-2$. It was found that the reaction with 5 -g incrementof hydrogen chloride was rapid and orderly. Substantially all the hydrogen chloride was transferred in this way; the residual gas in the weighed cylinder was then condensed directly in the reactor by cooling it with a liquid nitrogen bath. To react the last traces of hydrogen chloride, the reactor was allowed to warm to room temperature and, with all valves closed, was allowed to stand for at least $24 \mathrm{hr}$. Vigorous shaking of the reactor after removing it and its connections from the train also helped to complete the reaction.

Trimethylborane was separated from the reaction mixture by distillation. For this process the top of the reactor was equipped with a small distillation column R, which had a spiral of stainless steel wire as packing. During distillation, a liquid bath in container $\mathrm{D}$ was kept near $-40{ }^{\circ} \mathrm{C}$ to provide reflux, and the reactor $\mathrm{C}-3$ was kept near $0{ }^{\circ} \mathrm{C}$. Trap T-2 was cooled to about $-196{ }^{\circ} \mathrm{C}$ and the entire left side of the train (i.e., to the left of valve
6) was continuously evacuated. After a period of reflux, valve 6 was partially opened to allow distillation of a small portion of the trimethylborane into trap T-2. Then valve 6 was closed to return the column to total reflux. This process was repeated until all the trimethylborane was transferred.

During this process appreciable amounts of hydrogen, methane, and other gases, indentified by mass spectrometric analysis, were removed from the system while the trimethylborane was retained in trap T-2. After distillation was complete, T-2 was warmed to $-78.5{ }^{\circ} \mathrm{C}$ and the vapor pressure of the liquid at that temperature was measured directly with a mercury manometer. If it was not greater than $31 \mathrm{~mm} \mathrm{Hg}$, the sample was transferred directly to cylinder C-4. From preliminary work, it was found that hydrogen chloride was the material chiefly responsible for any pressure greater than $31 \mathrm{~mm} \mathrm{Hg}$ at $-78.5^{\circ} \mathrm{C}$. If the pressure was greater than 31 $\mathrm{mm} \mathrm{Hg}$, the trimethylborane was transferred back to the reactor and allowed to come to room temperature and remain at this temperature for an additional $24 \mathrm{hr}$. After this, the distillation process was repeated. This procedure was found to give material free of hydrogen chloride as determined by the mass spectrometric analyses.

In the first series of runs, a total of $180 \mathrm{~g}$ of trimethylborane in a purified form was prepared. The first preparation $(33 \mathrm{~g})$ had a purity of 99.7 mole percent, the second and third $(147 \mathrm{~g}$ ) were 99.9 mole percent pure, as determined by cryoscopiccalorimetric procedures [1]. An additional $176 \mathrm{~g}$ of trimethylborane was prepared later and was also found to be 99.9 mole percent pure. Infrared analysis [2] of the material showed that several bands pre- 
viously reported and ascribed to trimethylborane [5] were absent; these bands are presumed to have been caused by impurities.

Samples of the purified trimethylborane were stored at $-78{ }^{\circ} \mathrm{C}$ pending measurements of certain physical constants. Later, the samples in stainless steel cylinders were kept at room temperature for several weeks and their vapor pressures at $-78.5^{\circ} \mathrm{C}$ redetermined. No significant change in pressure was observed, indicating that the compound is stable at room temperature.

The purified trimethylborane has been used in other laboratories of this Bureau for measurement of infrared spectrum [2], heat capacity, heats of fusion, transition, and vaporization and vapor pressure [1]. At the Naval Research Laboratory it has been used in a study of electron mobility in $\mathrm{B}\left(\mathrm{CH}_{3}\right)_{3}$ [3] and as a neutron counting gas for proportional counters [4].

(Paper 66A1-140)

\section{References}

[1] G. T. Furukawa and R. P. Park, Heat capacity, heats of fusion, transition, vaporization, and vapor pressure of trimethylborane, $\mathrm{B}\left(\mathrm{CH}_{3}\right)_{3}$, presented before the Symposium, From Borax to Boranes, of the Division of Inorganic Chemistry, 133d Meeting of the American Chemical Society, San Francisco, California, April 15$18,1958$.

[2] J. E. Stewart, J. Research NBS 56, 337 (1956) RP2684

[3] G. A. Ferguson, Jr., and F. E. Jablonski, Rev. Sci. Instru. 28, 893 (1957).

[4] G. A. Ferguson, C. W. Peters, and F. E. Jablonski, An investigation of boron trimethyl counters, Progress Report of Naval Research Laboratories, March 1956 issue.

[5] G. Goubeau and H. J. Becher, Z. anorg. u. allgem. Chem. 268, 1 (1952).

[6] H. C. Brown, J. Am. Chem. Soc. 67, 374 (1945).

[7] H. C. Brown, H. Bartholomay, Jr., and M. D. Taylor, J. Am. Chem. Soc. 66, 435 (1944).

[8] A. R. Glasgow and G. S. Ross, Anal. Chem. 26, 2003 (1954). 